\section{Massive haemoptysis caused by congenital absence of a segment of inferior vena cava}

\author{
M H Ashour, S K Jain, K M Kattan, \\ A Karim El-Bakry, M Khoshim, \\ F M Mesahel
}

\begin{abstract}
A patient with congenital absence of a part of the inferior vena cava is described. This resulted in spontaneous rupture of a bronchial vein leading to massive haemoptysis.
\end{abstract}

\section{(Thorax 1993;48:1044-1045)}

Anomalies of the inferior vena cava are often discovered incidentally during imaging studies performed for other reasons, since patients with these abnormalities are usually asymptomatic. Haemoptysis as a presenting feature of interrupted inferior vena cava has not been previously reported. We describe a patient presenting with massive haemoptysis.

\section{Case report}

A 28 year old Saudi woman, 36 weeks into her second pregnancy, was admitted as an emergency with massive haemoptysis. She coughed up approximately $800 \mathrm{ml}$ of fresh blood over four hours. There was no history of chest pain, pneumonia, asthma, tuberculosis, bleeding disorders, cigarette smoking, stigma suggestive of hereditary telangiectasia, or previous haemoptysis. Her pregnancy had been uncomplicated to date. Physical examination was

\section{Division of Thoracic Surgery \\ $\mathrm{MH}$ Ashour \\ $S$ K Jain \\ K M Kattan \\ Division of General Surgery \\ A Karim El-Bakry \\ M Khoshim \\ Division of \\ Anaesthesia \\ F M Mesahel \\ Department of \\ Surgery, PO Box 7805 , Riyadh 11472, Saudi Arabia \\ Reprint requests to: Dr M Ashour \\ Received 7 July 1992 \\ Returned to authors \\ 16 September 1992 \\ Revised version received \\ November 1992}

Accepted 7 December 1992

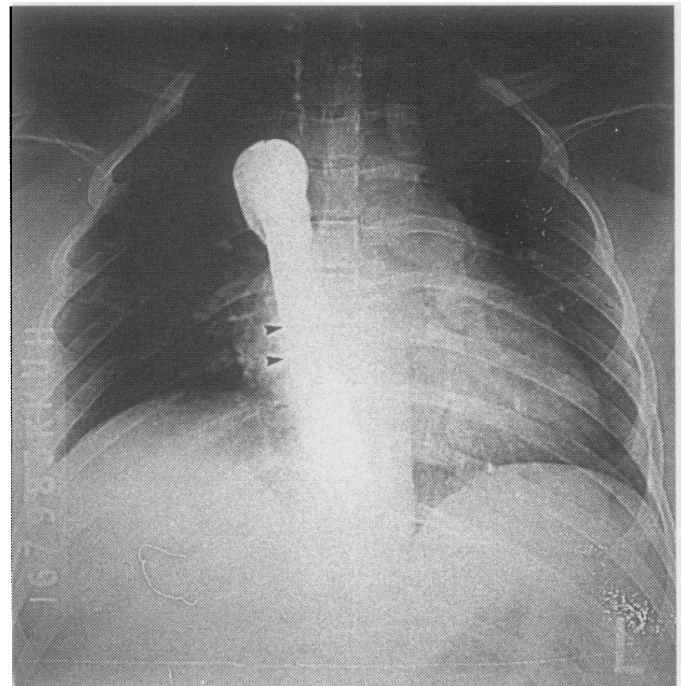

Figure 1 Early phase of pulmonary angiogram showing massively dilated azygos vein (arrows).

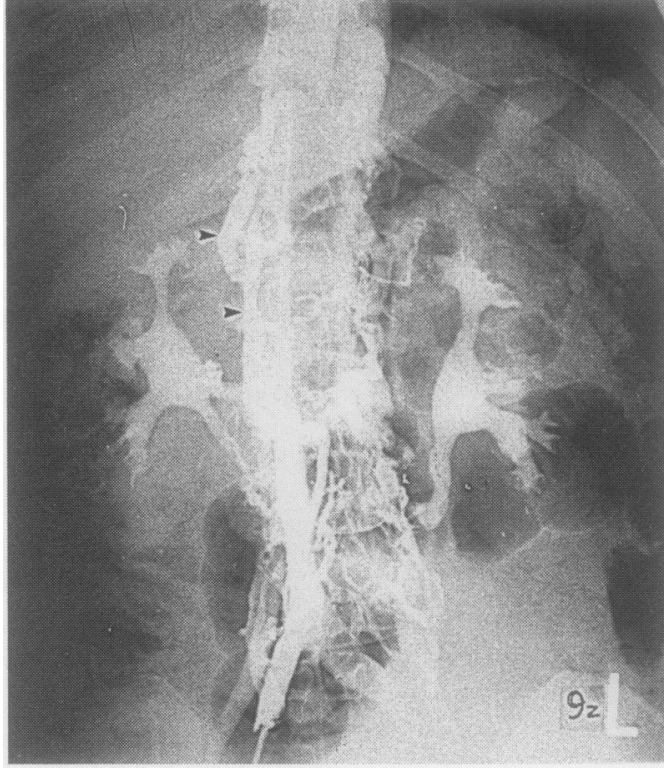

Figure 2 Cavogram showing venous return through the ascending lumbar veins (arrows) as an alternative to the absent prerenal segment of the inferior vena cava.

unremarkable except for a res-piratory rate of $30 /$ minute and poor air entry over the right lung. Fetal heart rate was $140 /$ minute.

Chest radiography showed a hyperinflated right lung with mediastinal shift to the left. Arterial blood tensions breathing room air showed a $\mathrm{pH}$ of $7 \cdot 41, \mathrm{PO}_{2}$ of $58 \mathrm{~mm} \mathrm{Hg}$, and $\mathrm{PCO}_{2}$ of $28.7 \mathrm{~mm} \mathrm{Hg}$. Blood biochemical tests were normal.

At bronchoscopy there was blood in the right main bronchus. The right bronchus intermedius was occluded by a clot-like material which could not be sucked out. A caesarian section was performed and a healthy baby was delivered. During this procedure another major haemoptysis occurred. At thoracotomy the azygos vein was found to be massively dilated. The bronchial as well as the intercostal veins were also dilated to 2-3 times their normal size. In view of the uncertainty about the pathological condition of the right bronchus intermedius, bronchotomy was performed rather than proceeding to lobectomy. A clot was seen to fill the bronchus. While removing the clot fresh bleeding from a small spot in the bronchial mucosa located immediately below the middle lobe orifice was seen. The pattern of bleeding was not arterial and the mucosa surrounding the area of bleeding looked normal. The bleeding site was transfixed with interrupted Dexon sutures. Postoperative recovery was uneventful.

Ten days after the operation, in search of a cause of the haemoptysis, the patient underwent further investigations. Pulmonary angiography showed no evidence of an arteriovenous fistula or pulmonary embolism. The contrast medium filled a dilated azygos vein that directed the flow to the superior vena cava (fig 1). A cavogram attempted from the femoral vein showed absence of the prerenal segment of the inferior vena cava (fig 2), with 
venous return through the ascending lumbar veins. The bronchial and intercostal arteries were normal on a thoracic aortogram. Echocardiography was normal. Computed tomography of the abdomen and chest showed no evidence of thrombosis in the inferior vena cava. The patient was followed up for 18 months and had no more episodes of haemoptysis.

\section{Discussion}

Although anomalies of the inferior vena cava are commonly discovered during imaging for other diseases, ${ }^{1-3}$ it seems that the anomaly in this patient directly contributed to the pathogenesis of the haemoptysis.

The bronchial venous system communicates freely with the pulmonary veins and alveolar capillaries on one side, and with the azygos vein on the other. ${ }^{4-6} \mathrm{~A}$ direct communication between the systemic venous circulation and the pulmonary circulation is therefore present. This makes the bronchial veins vulnerable to pressure changes in either the systemic venous or pulmonary circulations. These bronchial venous channels are thin walled and non-distensible. ${ }^{5}$ Physiologically about one third of the blood accumulated in the bronchial venous plexuses is thought to return to the azygos vein, while the remaining blood flow returns to the pulmonary veins. ${ }^{7}$

Although we did not measure pressure in the azygos vein, we believe that the congenital interruption of the inferior vena cava caused azygos venous hypertension due to the massive increase in the azygos venous flow. As a result of this haemodynamic change the bronchial venous drainage to the azygos vein could become impaired and reversal of bronchial venous flow might occur. Engorgement of the bronchial veins would therefore take place, similar to oesophageal varices in portal hypertension. These engorged, thin walled, relatively non-distensible, submucosal bronchial veins may undergo rupture with manoeuvres associated with increased intrathoracic pressure and this could lead to haemoptysis.

On the basis of our experience with this case we suggest that an inferior vena cavogram should be considered as one of the investigations when dealing with massive or recurrent haemoptysis of unknown origin.

We would like to thank Ms Tess M Formilleza for her help in setting up our manuscript.

1 Todisco T, Dottorini M, Parise P, Boschetti E, Lupatteli L. Inferior vena caval defects and the venous circulation. Chest 1987;92:768-9.

2 Hawass ND, Kolawole TM, Badawi MG. Concomitant pulmonary arteriovenous and inferior vena cava malformations: a case report. Eur $\mathcal{F}$ Radiol 1988;8:102-5.

3 Brem AS, O'Shea PA, Wesselhoeft CA. Absence of inferior vena cava associated with an atrophic right kidney and hypertension. F Urol 1981;126:539-41.

4 Tobin CE. The bronchial arteries and their connections with other vessels in the human lung. Surg Gynecol Obstet 1952;95:741-50.

5 Murata K, Itoh $H$, Todo G, Kanaoka $M$, Furata $M$, Torizuka $\mathrm{K}$. Bronchial venous plexus and its communication with pulmonary circulation. Invest Radiol 1986; 21:24-30.

6 Ohmichi M, Tagaki S, Nomura N, Tsunematsu K, Suzuki A. Endobronchial changes in chronic pulmonary venous hypertension. Chest 1988;94:1127-32.

7 Fraser RG, Pare JA. Diagnosis of diseases of chest. 2nd ed. Philadelphia: Saunders, 1978:83-5.

\section{Comparison of nebulised aerosol deposition in the lungs of healthy adults following oral and nasal inhalation}

\author{
Mark L Everard, John G Hardy, \\ Anthony D Milner
}

\section{Department of Child \\ Health \\ M L Everard \\ A D Milner \\ Department of Medical Physics \\ J G Hardy \\ Queens Medical \\ Centre, Nottingham \\ NG7 2UH \\ Reprints will not be available \\ Received 24 August 1992 \\ Returned to authors \\ 22 October 1992 \\ Revised version received \\ 20 November 1992 Accepted \\ 4 December 1992}

(Thorax 1993;48:1045-1046)

Although the nose acts as a filtration system it

\begin{abstract} thenetration of aero. Pen breathing through the nose compared with mouth breathing.
\end{abstract}

has been argued that patient preference should determine whether a facemask or a mouthpiece is used when inhaling aerosols from jet nebulisers. ${ }^{12}$ This argument is based on clinical studies which failed to show any significant difference in clinical response when salbutamol was inhaled through these two routes. ${ }^{13}$ However, bronchodilators are generally used in supramaximal doses ${ }^{45}$ and hence the observed clinical response need not directly reflect the total dose reaching the lungs.

The dose of aerosol deposited in the lungs is determined by the total dose of drug inhaled and the pattern of deposition of that dose within the airways. The factors influencing the total dose of drug inhaled when using a jet nebuliser are complex and have previously been discussed. ${ }^{6}$ The purpose of this study was to determine what effect inhaling a wet, heterodispersed aerosol by the nasal rather than the oral route might have on the pattern of deposition within the airways. A standard technique $^{57}$ was used in which the deposition of a radiolabelled aerosol was assessed with a gamma camera.

\section{Methods}

Eight men aged 21-32 years were studied. All were in good health, were non-smokers, and gave no history of lower respiratory tract dis- 